Safety of remote monitoring for patients taking androgen receptor antagonists

\title{
Time trends of drug-specific actionable adverse events among patients on androgen receptor antagonists: Implications for remote monitoring
}

Lauren Fleshner ${ }^{1}$; Alejandro Berlin ${ }^{2}$; Karen Hersey ${ }^{1}$; Miran Kenk ${ }^{1}$; Katherine Lajkosz ${ }^{1}$; Susan Nguyen $^{1}$; Jacob Wise ${ }^{1}$; Sophie O'Halloran ${ }^{1}$

${ }^{1}$ Division of Urology, University Health Network, University of Toronto, Toronto, ON, Canada; ${ }^{2}$ Department of Radiation Oncology, University Health Network, University of Toronto, Toronto, ON, Canada

Cite as: Fleshner L, Berlin A, Hersey K, et al. Time trends of drug-specific actionable adverse events among patients on androgen receptor antagonists: Implications for remote monitoring. Can Urol Assoc J 2021 October 18; Epub ahead of print. http://dx.doi.org/10.5489/cuaj.7437

Published online October 18, 2021

Corresponding author: Dr. Lauren Fleshner, Princess Margaret Cancer Centre, Toronto ON, Canada; lauren.fleshner@mail.utoronto.ca

$* * *$

\section{Abstract}

Introduction: In light of COVID-19, reducing patient exposure via remote monitoring is desirable. Patients prescribed abiraterone/enzalutamide are scheduled for monthly in-person appointments to screen for adverse events (AEs). We determined time trends of drug-specific actionable AEs among users of abiraterone/enzalutamide to assess the safety of remote monitoring.

Methods: A chart review was conducted on 828 prostate cancer patients prescribed abiraterone and/or enzalutamide. Data were collected to determine time to actionable first AEs, including hypertension, elevated liver enzymes (aspartate transaminase [AST], alanine transaminase [ALT]), hyperbilirubinemia, and hypokalemia. Survival analysis was used to determine time to AEs.

Results: In this study, 425 and 403 patients received enzalutamide and abiraterone, respectively. In total, $25.6 \%$ of those who took enzalutamide experienced an $\mathrm{AE}$, compared to $28.8 \%$ of patients on abiraterone. For patients using abiraterone and experiencing an AE, cumulative incidence of AEs at three, six, nine, and 12 months were: $67.2 \%, 81.9 \%, 90.5 \%$, and 93.9\%, respectively. Among enzalutamide users experiencing an AE, cumulative incidence of AEs at three, six, nine, and 12 months were $51.4 \%, 70.7 \%, 82.6 \%$, and $88.1 \%$, respectively. The AEs associated with enzalutamide were hypertension and liver dysfunction $(77.1 \%$ and $22.9 \%$, 
respectively). In the abiraterone group, associated AEs were liver dysfunction (47.4\%), hypertension (47.4\%), and hypokalemia (5.2\%).

Conclusions: Attaining AEs secondary to abiraterone/enzalutamide decreases over time and tends to occur within the first six months of therapy. Most actionable AEs can be remotely monitored. Given COVID-19, remote monitoring after six months of initiating abiraterone or enzalutamide appears appropriate.

\section{Introduction}

Prostate cancer is the most common malignancy among men in Canada, with an estimated 23,300 men being diagnosed with the disease in 2020. It is further estimated in 2020 that 4,200 men will die from prostate cancer - accounting for $10 \%$ of all cancer-related deaths in Canadian men [1]. The leading cause of death among metastatic prostate cancer patients is castrationresistant prostate cancer (CRPC).

Over the past ten years significant advances in managing patients with prostate cancer have been made, particularly due to the understanding of the androgen receptor axis as a major driver of prostate cancer physiology. Development of novel compounds targeting this axis have led to significant improvements in overall survival and prostate cancer specific outcomes.

Abiraterone and enzalutamide have been extensively studied and approved as hormonal therapies for patients with castration-resistant and castration-sensitive disease [2]. Oncologists and Urologists have gained considerable experience utilizing these therapies in clinical practice safely and efficiently in the outpatient setting [3].

Adverse events (AEs) associated with these medications have been thoroughly described [4][5]. Common AEs associated with abiraterone include hypertension, elevated serum liver enzymes, fluid retention and hypokalemia. In addition, enzalutamide also can cause fatigue, hypertension and (in rare cases) seizure activity. Drug-specific monographs as well as treatment guidelines recommend monthly evaluation for AEs associated with abiraterone. Periodic blood pressure monitoring is also recommended for enzalutamide users [6]. Our institutional practice for patients taking either abiraterone or enzalutamide is in-person monthly visits to monitor for AEs as well as disease progression.

In light of the COVID-19 era, reducing in-person visits via remote monitoring is desirable, in order to reduce patient and healthcare provider exposure. The purpose of this study is to investigate the real-world time trends of drug specific AEs, and to utilize these data to inform recommendations regarding remote monitoring of advanced prostate cancer patients undergoing treatment with abiraterone or enzalutamide. In particular, we strived to determine the safety among immediately actionable AEs such that emergent/urgent care would be required should they occur. Fatigue, for example, was not included in this study for this reason. 


\section{Methods}

A retrospective single institution chart review was conducted at the Princess Margaret Cancer Centre among patients diagnosed with advanced prostate cancer who were treated with abiraterone and/or enzalutamide. Patients in this cohort were treated between 2010 and 2020.

Among patients receiving abiraterone, $97.2 \%$ of patients were additionally prescribed $10 \mathrm{mg}$ prednisone for CRPC. Those receiving both abiraterone and enzalutamide (sequentially after progression) were treated as separate subjects. The adverse events documented included: hypokalemia, hypertension, and abnormal liver function tests. The liver function tests included alanine aminotransferase (ALT), aspartate aminotransferase (AST) and bilirubin. We chose these four anomalies as they are the most common AEs of special interest associated with these drugs, aside from fatigue. Edema and hypertension are highly correlated and both a manifestation of fluid overload. With respect to fatigue, we elected to omit this side effect as immediate medical attention is not required when present. The interval between commencement of the drug to the first $\mathrm{AE}$ was documented. If two or more AEs were identified on the same date, the most severe AE was recorded. It should be noted that patients are routinely seen Q4 weeks in the CRPC and Q12 weeks in the hormone sensitive prostate cancer (HSPC) setting. At each visit, LFTs, BP assessment and potassium are always measured. For this manuscript, we conducted a mini review of 50 patient visits and note that in 48 of the 50 , specific documentation was noted upon chart review. For patients who did not experience an adverse event, the end point was determined to be the last date of the patient taking the prescribed drug or the most recent date of follow-up. The AEs were graded according to the Common Terminology Criteria for Adverse Events, v5.0. For patients that had a grade $3 \mathrm{AE}$, subsequent AEs were recorded to determine if there was a correlation between first and second AEs. The Kaplan-Meier method was used to calculate time-to AE. Analyses were conducted using GraphPad Prism, v8.

\section{Results}

In total, 828 cases of abiraterone/enzalutamide treatment were identified, representing 672 unique patients (i.e. 156 patients [23.2\%] received both drugs during their disease treatment course). Among the 828 treatment regimens, 403 patients received abiraterone and 425 patients received enzalutamide. For those that were prescribed abiraterone, $28.8 \%$ experienced an AE ( 116 cases), compared to $25.6 \%$ in those utilizing enzalutamide (109 cases). Of the 225 combined AEs, 189 (84\%) patients experienced an AE during first-line treatment with either abiraterone/enzalutamide compared to $36(16 \%)$ AEs during sequential second-line treatment with either drug. Of the 156 patients that received both treatment regimens, 15 patients were reported to experience an AE on both treatments. Time-to-event analysis (Figure 1) revealed that patients were more likely to experience an $\mathrm{AE}$ receiving abiraterone compared to those on enzalutamide (HR 1.33, 95\% CI 1.03 to 1.73 , p-value 0.03 ). Sensitivity analysis was carried out among patients who did not experience both therapies and the results were unaffected (data not shown). 
For patients that experienced any AE during follow-up, $87.2 \%$ and $80.2 \%$ of said AEs occurred within the first 6 months of therapy for patients on abiraterone and enzalutamide, respectively (Figure 2 and 3 ).

For patients utilizing Abiraterone and experiencing an AE, cumulative incidence of AEs at 3,6,9 and 12 months were: $67.2 \%, 81.9 \%, 90.5 \%$ and $93.9 \%$, respectively. Among Enzalutamide users experiencing an AE, cumulative incidence of AEs at 3,6,9 and 12 months were: $51.4 \%, 70.7 \%, 82.6 \%$ and $88.1 \%$, respectively.

With regards to the severity of adverse events, the distribution of adverse events among grade $1,2,3$ and 4 were $32 \%, 44.9 \%, 22.7 \%$ and $0.4 \%$, respectively. There were no grade 5 AEs. The most commonly reported $\mathrm{AE}$ in the abiraterone group was abnormal liver function, grade 1 severity and in the enzalutamide group; hypertension, grade 2. Hypertension was the most commonly reported grade $3 \mathrm{AE}$ in both groups. One grade $4 \mathrm{AE}$ occurred in the abiraterone group, attributable to hypokalemia. No cases of hypokalemia were reported in the enzalutamide group. Of the 6 hypokalemia cases in the abiraterone group, all patients had been receiving 10mg prednisone at the time of the AE (Table 1).

Of the grade 3 and $4 \mathrm{AEs}$ on abiraterone, once resolved, 6 patients had a second $\mathrm{AE}$, following the first, on continuing treatment with abiraterone $(25 \%, 1.5 \%$ of the entire abiraterone cohort). Of the grade 3 AEs in the enzalutamide group, $4(14.3 \%, 0.94 \%$ of the entire enzalutamide cohort) experienced a subsequent $\mathrm{AE}$ on enzalutamide treatment. All of these second AEs were grade 1 liver function abnormalities and the first AE was hypertension in both groups.

\section{Discussion}

Abiraterone and enzalutamide are two frequently used drugs in the treatment of prostate cancer that require frequent and regular monitoring of AEs which to date have been conducted as inperson outpatient visits. This has been challenging in the current COVID-19 pandemic as frequent excursions to hospitals and healthcare facilities increases the risk for both patients and providers of contracting the virus. Furthermore, emerging data has suggested cancer patients harbor higher susceptibility and risk of contracting a more severe infection [7]. This study is relevant as we examined the frequency of AEs in a large population, with added health risks, thus making social distancing and reduction of potential exposure to COVID-19 a pressing need.

We found that over $80 \%$ of the first AEs occurred within 6 months of patients initiating abiraterone/enzalutamide. In addition, the majority of AEs were graded as grade 1 or 2 with only $22.7 \%$ made up of grade 3 AEs. Of the grade 3 AEs, hypertension was the most common event in both treatment groups. This suggests that if an $\mathrm{AE}$ is going to occur, it will likely be early in the course of treatment and is less likely to be a higher-grade AE. Implications of this finding suggest that monitoring of blood pressure, aminotransferases, bilirubin and potassium remotely in the community is reasonable after this time has elapsed, allowing a less taxing in-person follow-up in the outpatient hospital setting. A recent study assessing the safety profile of abiraterone in the mCRPC population found similar time-trends of adverse events. They 
suggested monitoring transaminases for the initial 5 months after commencing abiraterone, and kalemia and blood pressure monitoring for 7 months [8]. Although differences are noted between the two compounds, we emphasize that this should not be misinterpreted that one agent is safer than another, as numerous upstream factors (e.g: preexisting comorbidities) play a role in clinician selection of one particular compound.

Moreover, having a significant $\mathrm{AE}$ on either treatment is unlikely to be associated with the occurrence of a second $\mathrm{AE}$, as a small proportion of those that had a grade 3 or $4 \mathrm{AE}$ subsequently experienced a second. When second AEs did occur in this subset of patients, all were of grade 1 severity and resolved while continuing treatment, further supporting the hypothesis.

Limitations of this study include its retrospective nature. For example, it is plausible that patients who had significant AEs may not have presented to our hospital network and/or did not get reported at subsequent follow-up, which albeit plausible is most unlikely given the nature and set-up of the Canadian healthcare system. Also, we have not assessed the impact of remote monitoring on assessment of disease progression status, which is highly important moving forward to confirm the observations derived from this study. However, evaluation of PSA levels and imaging studies by remote surveillance have been successfully implemented in other institutions [9]. There is promising evidence supporting Telehealth improving cancer patients' quality of life [10], without compromising patient safety [11]. Furthermore, we did not specifically address leg edema as a possible AE associated with Abiraterone, although this could be addressed by telephone questioning, but would require further research.

In light of these findings, regular follow-up with in-person outpatient appointments in hospital for the first 6 months after abiraterone/enzalutamide appears appropriate. Thereafter, remote monitoring can provide a valuable alternative for patients and providers alike. Our observations are relevant during the current need for physical distance due to COVID-19, but also in the future to tailor and improve the value of cancer care in an increasingly virtual world.

\section{Conclusions}

Remote monitoring of AEs associated with abiraterone/enzalutamide treatment seems safe particularly after a 6-month period post treatment initiation with traditional monitoring. This approach can help decrease the risk of exposure to COVID-19 and improve the value of care for prostate cancer patients beyond the pandemic. 


\section{References}

1. Brenner DR, Weir HK, Demers AA, et al. Projected estimates of cancer in Canada in 2020. CMAJ Can Med Assoc J J Assoc Medicale Can. 2020;192(9):E199-E205.

2. Ryan CJ, Smith MR, Fizazi K, et al. Abiraterone acetate plus prednisone versus placebo plus prednisone in chemotherapy-naive men with metastatic castration-resistant prostate cancer (COU-AA-302): final overall survival analysis of a randomised, double-blind, placebo-controlled phase 3 study. Lancet Oncol. 2015;16(2):152-160.

3. Woon DTS, Finelli A, Cheung, DC et al. A population-based study comparing outcomes for patients with metastatic castrate resistant prostate cancer treated by urologists or medical oncologists with first line abiraterone acetate or enzalutamide. Urology. 2021 Feb 13;S0090-4295(21)00172-2.

4. Beer TM, Armstrong AJ, Rathkopf D, et al. Enzalutamide in Men with Chemotherapynaïve Metastatic Castration-resistant Prostate Cancer: Extended Analysis of the Phase 3 PREVAIL Study. Eur Urol. 2017;71(2):151-154.

5. Fukasawa S, Suzuki H, Kawaguchi K, et al. Efficacy and safety of abiraterone acetate plus prednisone in Japanese patients with newly diagnosed, metastatic hormone-naïve prostate cancer: a subgroup analysis of LATITUDE, a randomized, double-blind, placebo-controlled, Phase 3 study. Jpn J Clin Oncol. 2018;48(11):1012-1021.

6. Iacovelli R, Verri E, Cossu Rocca M, et al. The incidence and relative risk of cardiovascular toxicity in patients treated with new hormonal agents for castrationresistant prostate cancer. Eur J Cancer Oxf Engl 1990. 2015;51(14):1970-1977.

7. Liang W, Guan W, Chen R, et al. Cancer patients in SARS-CoV-2 infection: a nationwide analysis in China. Lancet Oncol. 2020;21(3):335-337.

8. Marret G, Doucet L, Hennequin C, Fizazi K, Culine S. Abiraterone in metastatic castration-resistant prostate cancer: Efficacy and safety in unselected patients. Cancer Treat Res Commun. 2018;17:37-42.

9. Frankland J, Brodie H, Cooke D, et al. Follow-up care after treatment for prostate cancer: evaluation of a supported self-management and remote surveillance programme. BMC Cancer. 2019;19(1):368.

10. Cox A, Lucas G, Marcu A, et al. Cancer Survivors' Experience With Telehealth: A Systematic Review and Thematic Synthesis. J Med Internet Res. 2017;19(1):e11.

11. Dickinson R, Hall S, Sinclair JE, Bond C, Murchie P. Using technology to deliver cancer follow-up: a systematic review. BMC Cancer. 2014;14:311. 


\section{Figures and Tables}

Fig. 1. Kaplan-Meier curve outlining the time to an adverse event for those treated with abiraterone (ABI) and enzalutamide (ENZA).

\section{Time to Adverse Event}

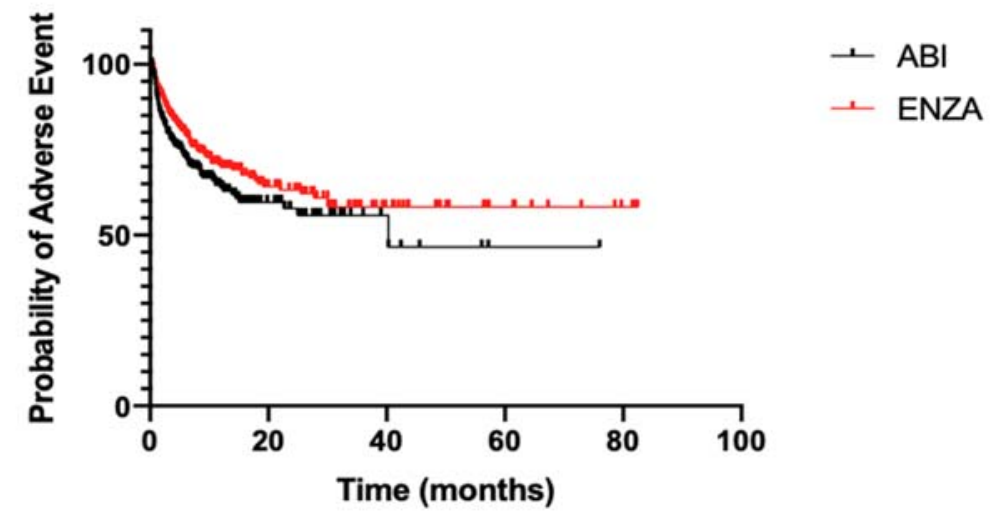

Fig. 2. Frequency of first adverse events in patients taking abiraterone for advanced prostate cancer.

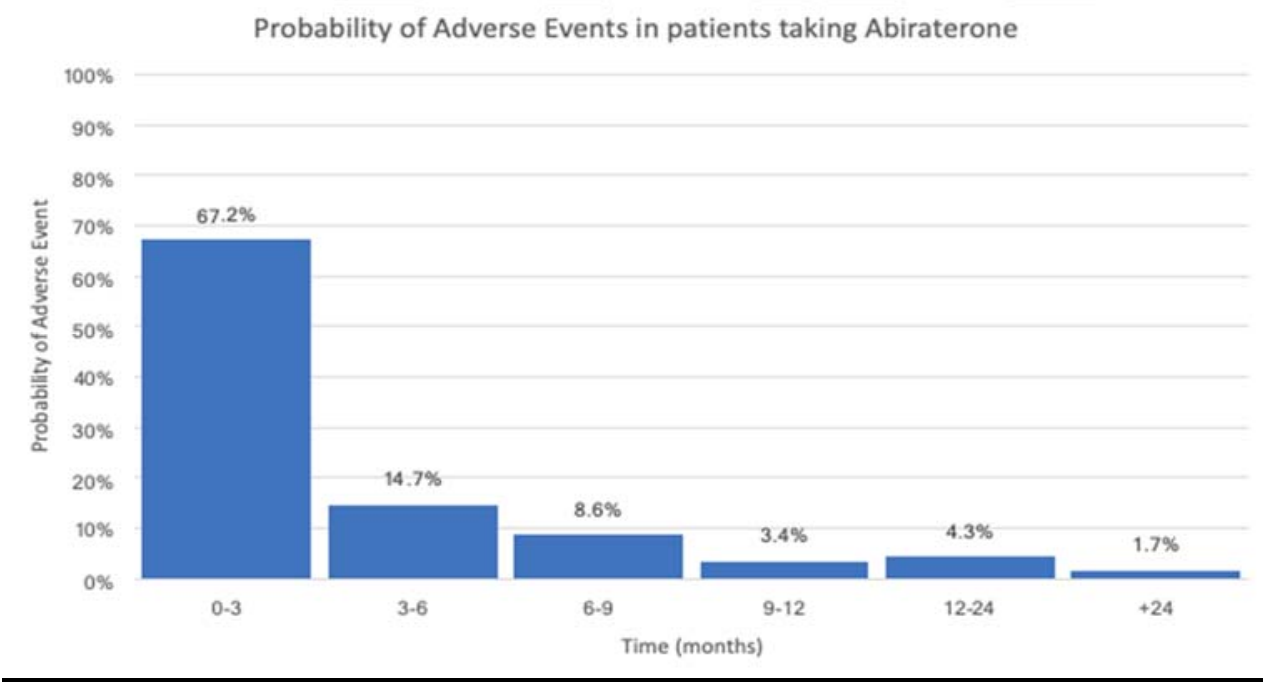


Fig. 3. Frequency of first adverse events in patients taking enzalutamide for advanced prostate cancer.

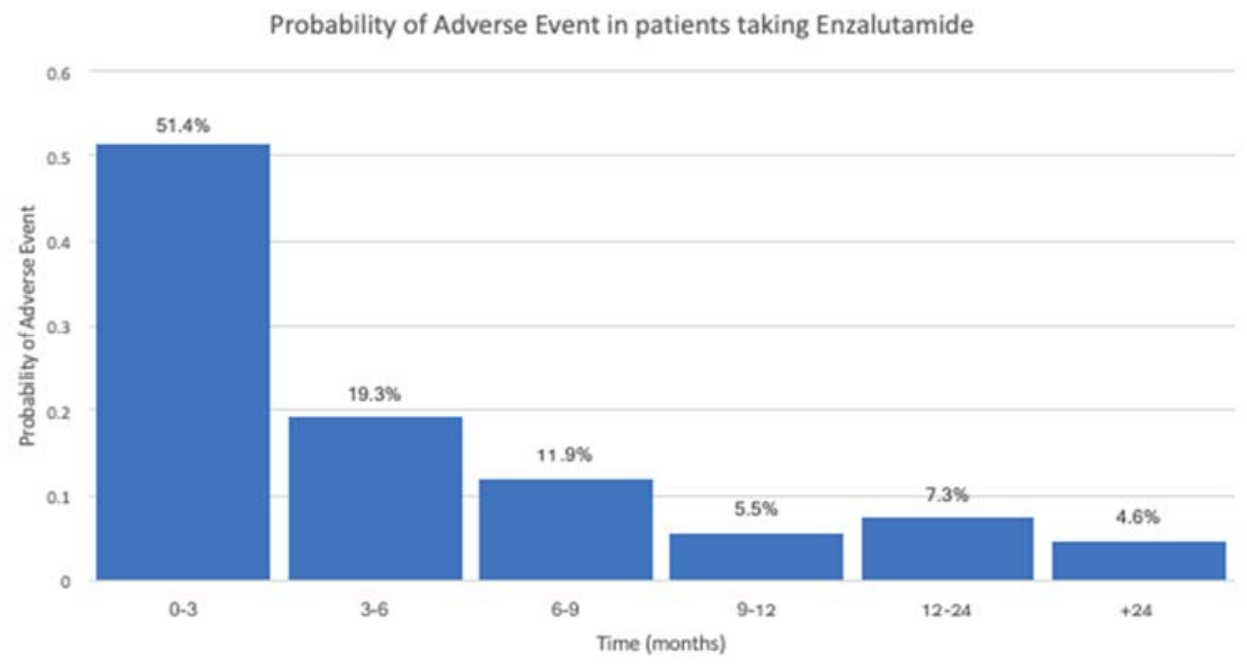

\begin{tabular}{|l|c|c|c|c|c|}
\hline \multirow{2}{|l|}{$\begin{array}{l}\text { Table 1. Distribution of CTCAE grades reported for patients taking abiraterone or } \\
\text { enzalutamide }\end{array}$} & \multicolumn{3}{|c|}{ Abiraterone } & \multicolumn{2}{c|}{ Enzalutamide } \\
\cline { 2 - 6 } & HTN & Hypokalemia & $\begin{array}{c}\text { Abnormal } \\
\text { liver } \\
\text { function }\end{array}$ & HTN & $\begin{array}{c}\text { Abnormal } \\
\text { liver } \\
\text { function }\end{array}$ \\
\hline CTCAE grades & Frequency & Frequency & $\begin{array}{c}\text { Frequency } \\
\text { Frequency }\end{array}$ & Frequency \\
\hline 1 & $4(3.4 \%)$ & $1(0.9 \%)$ & $40(34.5 \%)$ & $7(6.4 \%)$ & $20(18.3 \%)$ \\
\hline 2 & $37(31.9 \%)$ & 0 & $10(8.6 \%)$ & $49(45 \%)$ & $5(4.6 \%)$ \\
\hline 3 & $14(12.1 \%)$ & $4(3.4 \%)$ & $5(4.3 \%)$ & $28(25.7 \%)$ & 0 \\
\hline 4 & 0 & $1(0.9 \%)$ & 0 & 0 & 0 \\
\hline
\end{tabular}

CTCAE: Common Terminology Criteria for Adverse Events; HTN: hypertension. 